\title{
Molecular and cellular pathways involved in the therapeutic functions of MHC molecules; a novel approach for mitigation of chronic rejection
}

\author{
Thomas S. Skelton ${ }^{1}$, Malgorzata Kloc*, Rafik M. Ghobrial* \\ ${ }^{1}$ Department of Surgery, Methodist Hospital and Methodist Hospital Research Institute, Houston, USA. \\ *Department of Surgery, Methodist Hospital, Fannin St., Houston, USA; RMGhobrial@tmhs.org; mkloc@tmhs.org
}

Received 24 June 2011; revised 20 July 2011; accepted 28 July 2011.

\begin{abstract}
The mutated major histocompatibility complex (MHC) class I that contains donor-type epitopes displayed on recipient-type molecule was show$\mathrm{n}$ to inhibit acute and chronic rejection and induce indefinite survival of heterotopic cardiac allografts when administered in combination with a sub-therapeutic dose of cyclosporine (CsA) in a rat transplantation model. To elucidate the molecular pathways involved in the immunosuppressive effects of the mutated MHC molecule, we analyzed gene and protein expression profile during early and late phase following post-transplantation. Cytoskeletal structure analysis and expression status of Rho GTPase proteins, vacuolar transport and cytoskeleton regulatory pathways involved in immune response in $\mathrm{T}$ and dendritic cells demonstrated the novel mechanism for the abrogation of chronic rejection. Our studies confirm a new role of Rho GTPase pathway in the modification of $T$ cell motility and infiltration of the graft. We discuss these results within the framework of the most recent literature on MHC and molecular machinery controlling $T$ cell functions and dendritic cell antigen presentation.
\end{abstract}

Keywords: MHC; Transplantation; Allograft; Chronic Rejection; Rat

\section{INTRODUCTION}

The immune system plays a critical role in maintaining an individual's autonomy, and essential to its function is the ability to differentiate self from potentially harmful agents, such as microbial or viral proteins, foreign lipids and polysaccharides, which are viewed as non-self and are capable of eliciting an immune response. The transplantation of genetically incongruous organs produces an immunological response through allorecognition of nonself histocompatibility antigens. These antigens are located on cell surfaces and are capable of inducing an immune response in genetically dissimilar (allogeneic) recipients, resulting in graft injury and acute rejection of tissue or cells bearing non-compatible antigens.

Solid organ transplantation had been unsuccessful for many decades until the discovery in 1967 of the human major histocompatibility complex (MHC), which led to the introduction of human leukocyte antigens (HLA)matching method $[1,2]$. However, in spite of that discovery, patient and graft survival remained poor. Not until the 1980s, when the immunosuppressant cyclosporine became available for clinical use, did a significant improvement in transplant success rates appear $[3,4]$. Despite this discovery and the development of other novel immunosuppressants, better organ preservation, refined surgical techniques, and post-operative care, all of which help to eliminate acute rejection, chronic rejection continues to plague the majority of allografts and is a major obstacle for the long-term success of transplants. For instance, the one-year kidney allograft survival from cadaveric and living related donors has increased from $50 \%$ to roughly $90 \%$ and $95 \%$, respecttively, when compared with the allograft survival outcomes of 20 years ago, but the 10-year graft survival rates have fallen below $60 \%$, due to chronic allograft dysfunction $[5,6]$.

\section{MHC AND THE IMMUNE RESPONSE}

The rejection response elicited by transplantation between members of the same species is regulated through $\mathrm{T}$ and $\mathrm{B}$ cell recognition of tissues expressing genetically encoded polymorphisms within the MHC and the peptide fragments that they carry [6]. In humans, the MHC region resides on the short arm of chromosome 6 and contains more than 200 genes of which more than 40 known as the HLA genes, encode codominantly expressed 
cell surface proteins or components of the complement system. These genes and their translational products have been further grouped into three MHC classes, which are termed I, II, and III, based on their tissue distribution, structure, and function [7-9]. An important discovery in 1974 revealed that MHC molecules are functionally responsible for presenting peptide antigens to immune cells, thereby allowing the $\mathrm{T}$ cell receptor (TCR) to interact specifically with peptide fragments of a foreign protein bound within the peptide-binding groove of a MHC molecule [10]. Each MHC molecule consists of an extracellular peptide-binding cleft anchored to the cell by transmembrane and cytoplasmic domains. There is a high degree of allelic variation associated with the HLA system. For instance, there are 200 known variants of HLA-B, 500 variants of HLA-A, and over 1000 variants of HLA-DR [11,12]. Polymorphisms in the amino-acid sequence encoded by these alleles typically reside within or adjacent to the peptide cleft and facilitate antigen binding and presentation to $\mathrm{T}$ cells. Because of the amino acid variability in this region, each MHC binds an individual repertoire of protein antigens that are recognized by distinctly different $\mathrm{T}$ cells. The ability to potentially present a wide range of peptides on MHC molecules is the primary cause of HLA polymorphism and may provide a balance between maximizing the immunological response against invading antigens while preventing self-recognition and autoimmunity [13]. MHC class I and class II molecules both use very similar peptide binding domains, and peptide binding plays an integral role in the proper folding of the final peptide-MHC complex [14]. Peptide antigens are held in place by non-covalent interactions between the peptide and binding cleft, which determines peptide specificity, and a network of hydrogen bonds that determine the length of bound peptide [15]. Given the role of MHC molecules in antigen presentation, in the case of transplantation, allogeneic MHC molecules can act as both antigen-presenting molecules and as foreign antigens capable of eliciting an immune response [13].

Class I MHC is present on all nucleated cells and is composed of a 45-kd $\alpha$ heavy chain encoded by genes of the HLA-A, HLA-B, and HLA-C loci on chromosome 6 in association with a $12-\mathrm{kd}$ protein, $\beta_{2}$-microglobulin, which is encoded by a gene on chromosome $15[2,9]$. Class II MHC is expressed by professional antigen presenting cells (APC), namely, dendritic cells (DCs) and by $\mathrm{B}$ lymphocytes, macrophages, endothelial cells, and thymic epithelial cells [15]. Class II MHC is a heterodimer composed of non-covalently associated $\alpha$ and $\beta$ chains of approximately 230 amino acids, each encoded by the HLA-DR, HLA-DP, and HLA-DQ genes' loci [2].
Class III MHC genes encode components of the complement system and will not be discussed in this review. $\mathrm{CD}^{+}$cytotoxic $\mathrm{T}$ cells bind preferentially to class I $\mathrm{MHC}$ molecules and $\mathrm{CD}^{+}{ }^{+}$helper T cells bind to class II MHC molecules.

\section{MHC AND ANTIGEN PRESENTATION PATHWAYS}

Three unique, but not mutually exclusive, pathways of antigen presentation, including the direct, indirect, and semi-direct pathways, are used by MHC class I and class II molecules for the presentation of peptide antigens to $\mathrm{CD}^{+}$and $\mathrm{CD}^{+} \mathrm{T}$ cells, respectively, in the presence of transplanted tissue (Figures 1-3).

Transplantation of a solid organ from a genetically identical (syngeneic) individual does not lead to an immunological response against the graft. Disparities between self and non-self major histocompatibility complex (MHC) molecules, as in the context of genetically disparate (allogeneic) individuals, result in the activation of the innate immune response, as well as the initiation of an adaptive immune response, which subsequently leads to graft rejection and possible patient death $[6,15]$. Adaptive immunity involves allorecognition of allogeneic or foreign MHC antigens. An alloresponse to the graft occurs through the presentation of donor-specific antigens to recipient $T$ cells by engagement of a receptor-ligand system between the $\mathrm{T}$ cell receptor (TCR) and a foreign peptide-MHC complex. Following TCR ligation, additional co-stimulatory signals lead to $\mathrm{T}$ cell activation, proliferation of allospecific $\mathrm{T}$ cells, and recruitment of effector leukocytes to the graft itself $[13,16]$. This specificity depends on the recognition of short peptide antigens bound to polymorphic MHC-encoded glycoproteins on the cell surface of APCs and the antigenic nature of the MHC molecules themselves [6,17]. Additionally, B lymphocytes participate in the humoral response by presenting antigen to $\mathrm{CD}^{+} \mathrm{T}$ cells, which subsequently help B cells differentiate into all- antibody-producing plasma cells.

\section{DIRECT PATHWAY}

The direct antigen-presentation pathway, or the endogenous class I pathway (Figure 1(a)), is active in nearly all cells and consists of mechanisms for displaying peptides produced within cells in the context of class I MHC. This method of antigen presentation allows for the internal proteome to be sampled and immunologically surveyed by cytolytic $\mathrm{CD} 8^{+} \mathrm{T}$ cells, which have the ability to kill cells expressing viral proteins or tumor antigens [15]. During class I presentation, the MHC class I heavy chains assemble with the $\beta_{2}$-microglobulin fol- 


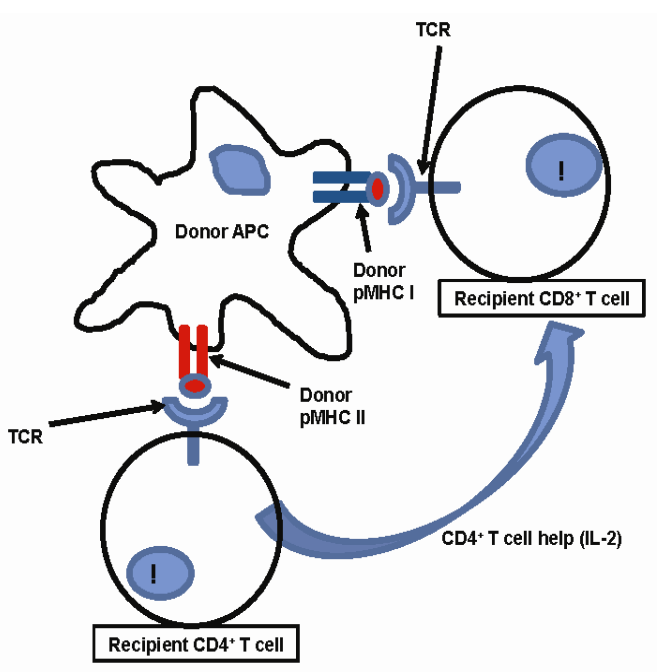

Donor derived APCs prime recipient $\mathrm{CD} 4^{+}$and $\mathrm{CD} 8^{+} \mathrm{T}$ cells by direct presentation of intact donor peptide-MHC complexes (pMHC). $\mathrm{CD}^{+} \mathrm{T}$ cells then provide helper signaling for cytotoxic $\mathrm{CD} 8^{+} \mathrm{T}$ cells primed by the same APC.

(a)

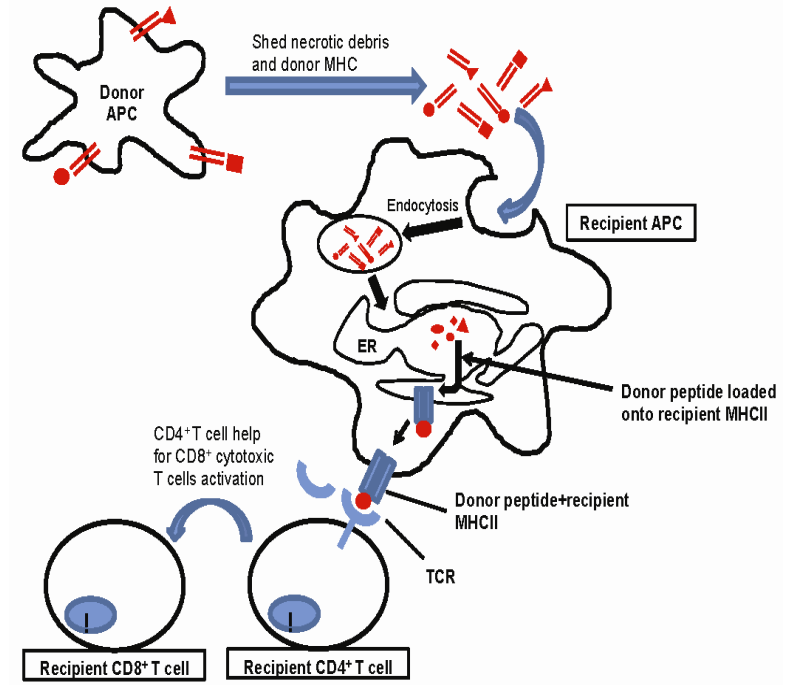

Recipient APCs phagocytose foreign MHC debris and present the processed peptides on recipient MHC II to class II restricted $\mathrm{CD}^{+} \mathrm{T}$ cells.

(b)

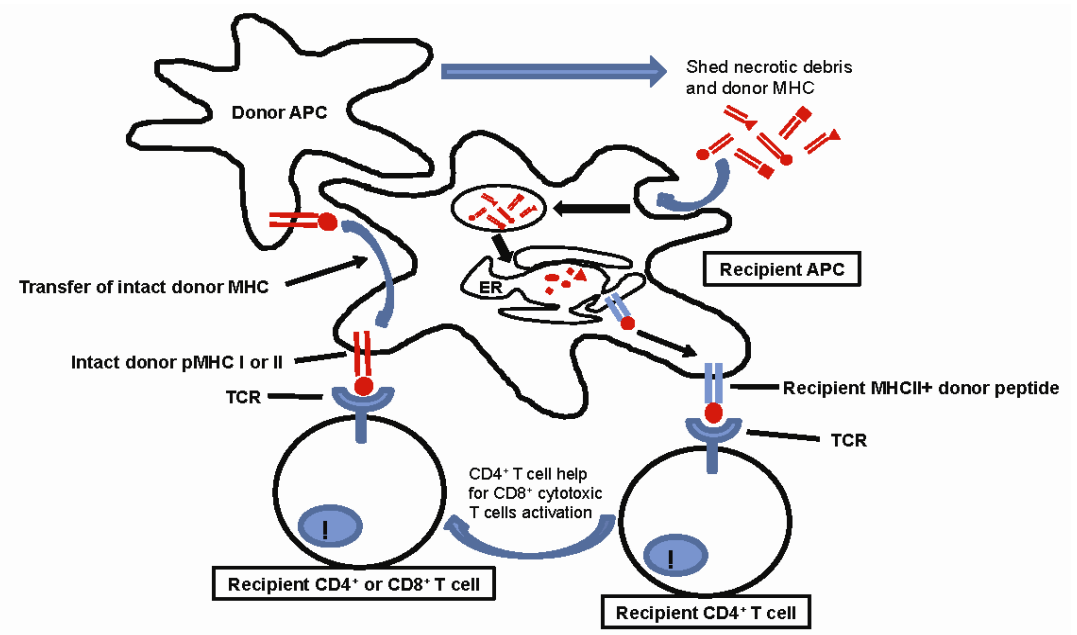

Intact donor class I or II MHC can be transferred to recipient APCs through cell-to-cell contact or exosomal vesicles. These, now chimeric, APCs can directly present intact donor peptide-MHC I or II (pMHC) to recipient $\mathrm{CD} 8^{+}$and $\mathrm{CD}^{+} \mathrm{T}$ cells, respectively, as well as indirectly present processed donor MHC to recipient class II restricted $\mathrm{CD} 4^{+} \mathrm{T}$ cells.

(c)

Figure 1. (a) Direct antigen presentation. (b) Indirect antigen presentation. (c) Semi-direct antigen presentation.

lowed by assembly of the peptide loading complex within the endoplasmic reticulum (ER) where endogenous peptides generated through the action of peptidases are delivered by the heterodimeric transporter associated with antigen processing (TAP). These peptide-MHC complexes are then transported to the Golgi for final processing and finally delivered to the cell surface for presentation (Figure 1(a); Figure 4) [18]. In the context of transplantation, direct presentation relies on the allorecognition of passenger leukocytes, specifically, the recognition of dendritic cells expressing intact surface class I or class II MHC, by recipient $\mathrm{CD}^{+}$and $\mathrm{CD}^{+} \mathrm{T}$ cells, respectively (Figures 1-2). This recognition primes $\mathrm{CD} 8^{+}$and $\mathrm{CD} 4^{+} \mathrm{T}$ cells. Next, the $\mathrm{CD}^{+}$cells are able to provide additional stimulatory signals for the differentiation of allospecific effector $\mathrm{CD}^{+}$cells that can wreak havoc upon the graft $[16,19]$. The presence of such donor APCs in the circulation following organ revascularization was demonstrated by Lechler and Batchelor [20], who showed reduced failure rates of rat renal allografts following transplantation of the graft in an intermediately immunosuppressed recipient, thereby depleting the graft of DCs before the final transplantation. 


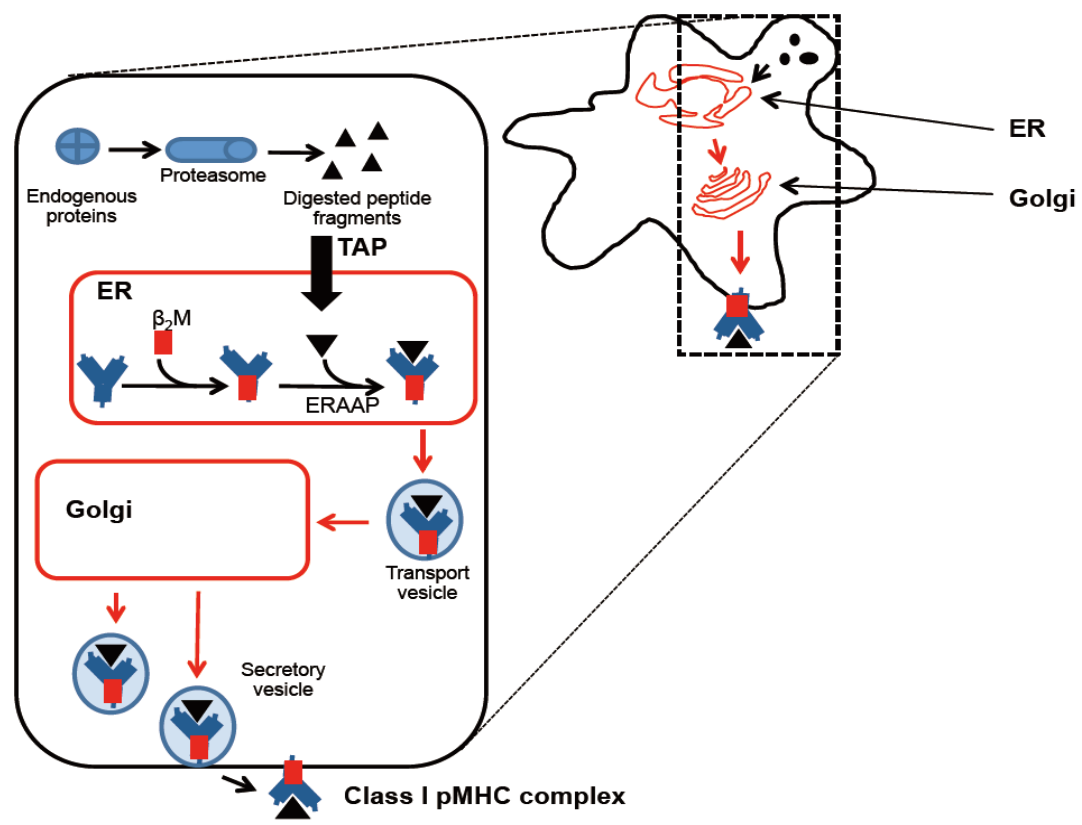

Endogenous proteins are processed through proteasomal peptidases, and are transported via TAP into the ER. MHC I heavy chains assemble with $\beta_{2}$-microglobulin $\left(\beta_{2} \mathrm{M}\right)$ within the ER. Endoplasmic reticulum aminopeptidase (ERAAP) mediates final peptide trimming before or after loading into the MHC peptide-binding groove. Secretory vesicles transport peptide-MHC I complex to the Golgi apparatus for final processing. Subsequently, the complex is transported to the cell surface via exosomes (secretory vesicles) for presentation to $\mathrm{CD} 8^{+} \mathrm{T}$ cells. The red colored portion of the diagram represents stages in antigen processing affected by allochimeric MHC treatment (see text).

Figure 2. Class I antigen processing.

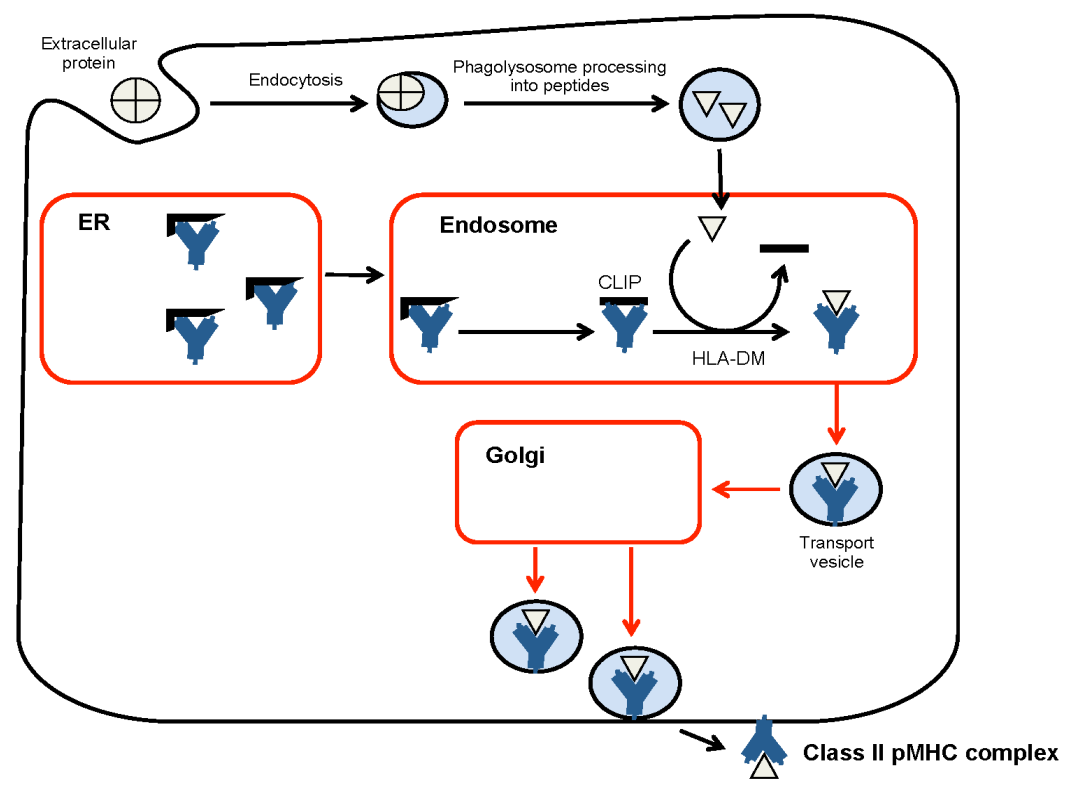

Extracellular antigen is endocytosed and digested within endocytic vesicles and transferred to the endosomal compartment where it is loaded into the protein-binding groove of class II MHC. MHC II was previously assembled in the ER in combination with its invariant chain (II), which is partially cleaved in the endosomal compartment leaving only the MHC class II-associated invariant-chain peptide (CLIP). HLA-DM catalyzes the substitution of CLIP for the processed extracellular peptide antigen and the class II pMHC complex is then transported to the Golgi and ultimately to the cell surface via exosomes (secretory vesicles) for presentation to $\mathrm{CD}^{+} \mathrm{T}$ cells. The red colored portion of the diagram represents stages affected by allochimeric MHC treatment (see text).

Figure 3. Class II antigen processing. 


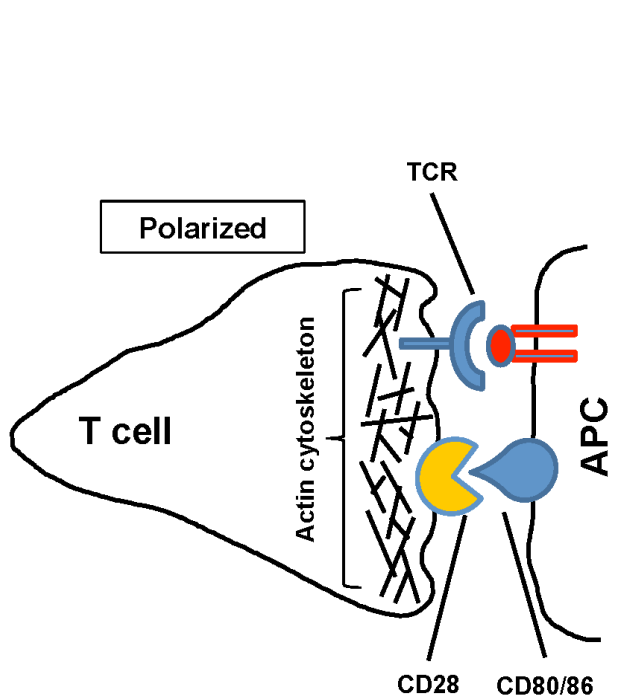

(a)

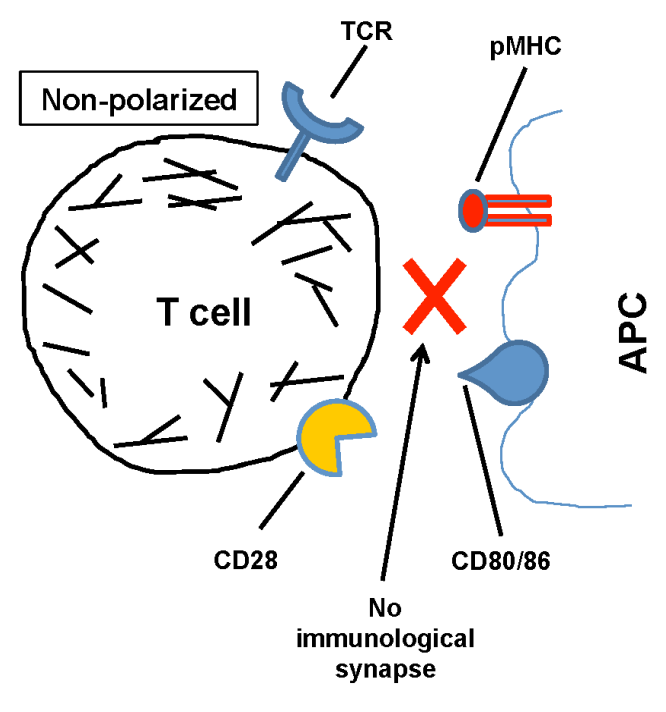

(b)

Figure 4. Actin cytoskeleton and formation of the immunological synapse (IS). (a) Actin cytoskeleton polymerization allows T cell polarization and motility, bringing TCR and co-stimulatory receptor, CD28, into the proper spatial and temporal orientation to facilitate IS formation between TCR-pMHC and CD28-CD80/86. (b) Treatment with allochimeric MHC (see text) results in dysregulation of actin cytoskeleton. This leads to failure in T cell polarization, IS formation, T cell motility and graft infiltration (see text).

During $\mathrm{T}$ cell development, thymic education ensures the selective survival of those lymphocytes capable of recognizing self MHCs. This process enables the mature $\mathrm{T}$ cell repertoire to become restricted to the recognition of foreign peptides bound to self MHCs, tolerant of self peptides, and adept at mounting an immune response toward foreign peptides through the process of positive selection, while cells bearing self-reactive TCRs are deleted through the process of negative selection [21]. Accordingly, it is surprising that the T cell repertoire contains such a high frequency of direct anti-donor reactiveity. It is unclear why, in the context of such MHC restriction, recipient $\mathrm{T}$ cells should ever recognize the allogeneic MHC molecules that are present in genetically incongruous allografts or mixed lymphocyte reactions. It has been shown that up to $10 \%$ of the $\mathrm{T}$ cell pool can react with intact allogeneic class I or II MHC expressed by passenger DCs that enter the circulation through the transplantation of vascularized grafts [22-24]. This paradox can only be explained by significant TCR crossreactivity between self-MHC and allogeneic MHC-peptide complexes. Two of the models proposed to account for this high frequency of alloreactive $T$ cells in the direct pathway are the "high determinant density" model and the "multiple binary complex" model $[25,26]$ in which either alloreactive $\mathrm{T}$ cells directly recognize exposed amino acid polymorphisms on intact allogeneic MHC molecules or differences in the allo-MHC peptide binding groove result in presentation of a set of peptides different from those of the self-MHC homologue [27,28]. The direct pathway dominates the early postoperative period and the acute rejection response but, despite the high frequency of allo-MHC specific T cells, it gradually declines with the depletion of donor APCs. The studies of Pietra et al. [29] provide additional evidence for the role of the direct pathway in allograft rejection. Both immunodeficient mice with severe combined immunodeficiency and recombination-activating-gene double negative (Rag-/-) mice, when reconstituted with singeneic $\mathrm{CD}^{+} \mathrm{T}$ cells, were able to reject class II MHCs expressing cardiac allografts but had limited rejection of grafts taken from MHC class II-deficient mice. Additionally, Rag-/- MHC II-/- mice receiving allogeneic cardiac grafts were fully capable of rejecting such grafts when reconstituted with $\mathrm{CD}^{+} \mathrm{T}$ cells [29]. While the direct pathway can directly activate $\mathrm{CD} 8^{+} \mathrm{T}$ cells, the importance of $\mathrm{CD}^{+} \mathrm{T}$ cells cannot be overstated, given that these mice lacked $\mathrm{CD} 8^{+} \mathrm{T}$ cells and the capability to generate an indirect response.

\section{INDIRECT PATHWAY}

In contrast, the indirect pathway of antigen presentation, or the exosomal class II pathway, involves endocytosis of extracellular antigens by recipient APCs. Akin to conventional antigen presentation for nominal antigens, following transplantation, host APCs migrate through the graft, picking up soluble donor MHCs, apoptotic cells and necrotic debris, all of which are internally processed and presented to recipient $\mathrm{CD}^{+} \mathrm{T}$ cells as peptide fragments within the peptide binding groove of self-class II MHC molecules (Figure 1(b)) [20,24,30,31]. Class II MHC assembly occurs initially in the ER in association with a transmembrane chaperone protein 
invariant chain. Within the endosomal compartment a series of protease cleave the invariant chain, leaving an MHC class II-associated invariant chain peptide (CLIP). Subsequently, the intracellular protein HLA-DM catalyzes the removal of CLIP and the loading of endocytosed peptides, or the peptide exchange, before their transport to the cell surface for immunologic presentation (Figure 1(b); Figure 3) [11,17,32,33]. Studies in mice suggested a role for the indirect antigen presentation pathway in transplant rejection through the presentation of allogeneic MHC by self-class II MHC H. DCs from $\mathrm{H}-2 \mathrm{Ab}$ recipients, which lack the $\mathrm{H}-2 \mathrm{E}$ antigen, stained positive for $\mathrm{H}-2 \mathrm{E}$ antigen after recipient injection with $\mathrm{H}-2 \mathrm{E}$ expressing $\mathrm{H}-2 \mathrm{k}$ cells [34]. Furthermore, CD8 ${ }^{+}$depleted or MHC class I-deficient mice receiving class II MHC deficient skin grafts rejected their grafts through the presentation of donor class I MHC on re- cipient class II molecules [35]. The pathways for cellular peptide antigen processing and their subsequent presentation involve an elaborate multistep process consisting of numerous cytosolic vesicles and organelles as well as a number of catalytic and degradation enzymes that function in peptide cleavage and loading into the protein binding groove of the MHC [36]. The gradual decline in direct alloreactivity, as donor APCs are depleted, is evident in grafts both with and without signs of chronic rejection, indicating that direct presentation does not play a major role in the development of chronic rejection. In contrast, indirect antigen presentation, by virtue of requiring the processing and presentation of endocytosed proteins, is a slower process and persists for as long as the graft is present, highly contributing to the development of chronic rejection [37]. The indirect antigen presentation pathway has been shown to be sufficient for the development of chronic allograft vasculopathy and arteriosclerosis in heart transplant models [38]. The requirement of antigen processing and presentation by recipient APCs in the context of self-class II MHC signifies that the indirect pathway of antigen presentation is largely dominated by $\mathrm{CD}^{+} \mathrm{T}$ cells [6]. However, some overlap exists, as alloreactive $\mathrm{CD}^{+}{ }^{+} \mathrm{T}$ cells can directly respond to intact class II $\mathrm{MHC}$, and alloreactive $\mathrm{CD}^{+} \mathrm{T}$ cells can be indirectly activated through "cross priming" which involves recipient APCs presenting donor antigens shed by surrounding cells in the context of self-class I MHC molecules [39]. The significance of the role that these indirectly responding $\mathrm{CD}^{+} \mathrm{T}$ cells play in facilitating chronic rejection remains unclear, but the importance of the indirect pathway as a whole has been well documented. While the majority of studies focus on the interaction between APCs and responding T cells, evidence also exist for the ability of recipient-derived endothelial cells to utilize indirect antigen presentation to promote further the rejection cascade. The endothelium of a transplanted graft is gradually replaced with recipient cells capable of presenting peptide in the context of class II MHC [34]. Targeting of these replaced cells by allo-reactive $\mathrm{T}$ cells provides further insight into the role of the indirect pathway in the development of the characteristic vascular lesions associated with chronic rejection [40].

\section{SEMI-DIRECT PATHWAY}

Recently, an additional mechanism of antigen presentation, known as semi-direct allorecognition has been uncovered (Figure 1(c)) [41]. The traditional model of cross talk between $\mathrm{CD}^{+}$and $\mathrm{CD}^{+} \mathrm{T}$ cells involves a linked "three cell" system in which the generation of antigen-specific $\mathrm{CD}^{+}$effector T cells by APCs requires additional stimulatory signaling from helper $\mathrm{CD}^{+} \mathrm{T}$ cells activated by the same APC [42]. Transplantation reveals limitations in this "three cell" model and suggests the presence of an unlinked "four cell" model in which crosstalk between directly activated $\mathrm{CD} 8^{+} \mathrm{T}$ cells relies on amplifying signals from indirectly activated $\mathrm{CD}^{+} \mathrm{T}$ cells stimulated by completely different APCs $[43,44]$. Immunological cells have the capability to exchange surface molecules through either cell-cell contact or through exosomes [45,46]. Semi-direct allorecognition therefore resolves the "four cell" conundrum by stipulating that recipient DCs can acquire and present intact donor class I or II MHC directly to $\mathrm{CD}^{+}$or $\mathrm{CD}^{+}$ $\mathrm{T}$ cells while maintaining the ability to internalize, process and present donor $\mathrm{MHC}$ as peptides indirectly to $\mathrm{CD}^{+} \mathrm{T}$ cells (Figure 3 ). In this manner, both direct and indirect antigen presentation occurs in a three cell manner involving the $\mathrm{DC}, \mathrm{CD} 4^{+} \mathrm{T}$ cells, and $\mathrm{CD}^{+} \mathrm{T}$ cells $[6,41]$. The transfer of intact donor MHC could be a means of continual direct antigen presentation in the face of diminishing donor APCs. Although no evidence has been identified for the in vivo occurrence of this mode of antigen presentation regarding allograft rejection, the semi-direct pathway could provide an alternative explanation for several scenarios that cannot be explained by only considering direct and indirect pathways. One example of these scenarios is the acute rejection of injected embryonic thymic epithelium in the absence of indirect presentation. Devoid of DCs, this response presumably occurs through the acquisition of donor thymic antigens presented directly to recipient $\mathrm{CD}^{+} \mathrm{T}$ cells [47].

\section{ALLOGRAFT TOLERANCE}

Large-scale efforts in clinical research and basic science have been devoted to unraveling the molecular basis of T-cell allorecognition, allograft rejection, and the 
development of allograft tolerance without the need for chronic immune suppression. Allorecognition by any of the previously mentioned pathways can ultimately lead to the activation and recruitment of allospecific $\mathrm{T}$ cell clones capable of producing aninflammatory response in the graft. However, the outcome of allorecognition is not as clear-cut as once thought. Allorecognition, in transplantation is also capable of inducing a state of graft acceptance, or tolerance. Allograft tolerance has been observed in numerous allogeneic animal transplant models across class I/II MHC barriers as well as in humans, especially following liver transplantation [48] in which the liver has an inherent ability to resist rejection through the expression of class I and II MHCs. This type of protective allorecognition is also evident during pregnancy where semi-allogeneic fetal tissue, consisting of both paternal and maternal antigens, is present yet the fetus is not rejected. These disparities allow a distinction to be made between antigenicity and immunogenicity, or the ability to recognize a foreign substrate and the ability to produce an immune response to eliminate it.

The development of immune tolerance involves the deletion of a large proportion of $\mathrm{T}$ cells with direct allospecificity and the continual suppression of remaining direct and indirect alloreactive cells that continue to be primed for the life of the graft. Many cells with such suppression or regulatory activity have been described in both mice and humans. The most studied of them are the $\mathrm{CD}^{+} \mathrm{CD}^{2} 5^{+} \mathrm{T}$ regulatory cells (Tregs), which express high levels of the transcription factor Foxp3 [49]. The development of Tregs occurs through the indirect pathway after recipient dendritic cells have migrated to the peripheral lymphoid tissue. The dependence on this pathway has been recently shown in rats in which depletion of host APCs caused the abrogation of tolerance in Lewis liver allografts in Brown Norway recipients [50]. The role of indirect antigen presentation in antibodymediated rejection is also influenced by Tregs. The rejection of class I disparate cardiac allografts in rats has been shown to be antibody-mediated and driven by indirect $\mathrm{T}$ cell help, which was abrogated following tolerizing protocols that induced specific $\mathrm{CD} 4^{+}$regulatory cells [51].

\section{MHC AS THERAPEUTIC AGENTS}

Studies looking at the potential for MHC to serve as immunomodulating agents have been underway for years, sparked by the work of Billingham, Brent and Medewar, who showed donor-specific tolerance to skin grafts produced by exposing recipients to donor antigens during fetal life [52]. Since then numerous studies investigating the therapeutic value of MHC molecules have been performed. Immunization with allogeneic peptides, eliciting only an indirect response is sufficient to propagate transplant rejection. However, hyporesponsiveness of indirectly allo-specific $\mathrm{T}$ cells can be achieved through intrathymic injection of MHC peptides [35,53]. Synthetic peptides corresponding to specific HLA sequences, specifically those within the $\alpha 1$ helix of HLA-A have been shown to inhibit cytotoxic $\mathrm{T}$ cell proliferation, produce immunological tolerance and prolong survival in animal transplant models. This peptide therapy was licensed under the brand name Allotrap and showed inhibition of cell-mediated immune responses to kidney allografts in phase II trials $[54,55]$. Synthetic peptides based on the $\alpha 1$ helix of HLA class II molecules similarly blocked T cell proliferation [55]. Although all of these studies indicate the potential therapeutic value of MHC molecules, the mechanisms underlying their immunomodulatory effects remain largely elusive.

\section{MOLECULAR AND CELLULAR PATHWAYS INVOLVED IN INHIBITION OF CHRONIC REJECTION BY MHC}

We have previously produced donor-specific transplantation tolerance in a rat heterotopic cardiac transplant model through the pre- and peri-operative administration of an allochimeric class I MHC. Dominant amino-acid epitopes identified on the $\alpha 1$ helix of the donor $\mathrm{MHC}$ were inserted into the $\alpha 1$ helical region of the recipient class I $\mathrm{MHC}$, creating the allochimeric construct [ $\alpha 1$ 1/u]-RT1.Aa [56]. By delivering this protein, in combination with sub therapeutic doses of cyclosporine (CsA) through the portal vein at the time of transplantation, we achieved donor specific tolerance, the attenuation of acute and chronic rejection, and prolongation of graft survival [56]. These observations have led our laboratory to investigate the molecular and cellular mechanism underlying the immunosuppressive effects of soluble allochimeric class I MHC.

Heterotopic cardiac transplantation was performed between donor Wistar Furth (WF) rats and ACI recipeents. Recipient animals were either untreated, treated for 7 days with therapeutic CsA, or treated with 3 days of sub-therapeutic CsA plus a single intraoperative dose of allochimeric class I MHC molecule delivered through the portal vein. Animals that received no immunosuppressant or received only sub-therapeutic CsA rejected their grafts in 5.4 or 16.2 days, respectively, whereas grafts from animals treated with combined low dose CsA and allochimeric peptide survived indefinitely [56]. Microarray analysis of the gene expression profile of heart allograft tissue and splenic $\mathrm{T}$ cells, demonstrated that allochimeric class I MHC therapy caused increase in expression of genes involved in the structural integrity of the heart muscle, Nexilin and Myocardin, at day 1 and 3 
post-transplantation compared with acutely rejecting controls, as well as downregulation in the pro-inflammatory cytokine IL- $1 \beta$ within the cardiac allograft [57]. There was also decreased expression of the partitioning defective 6 homologue beta (PAR6) gene, which functions in cell polarity and motility [57]. In addition, genes that play a role in actin filament polymerization, including RhoA and Rac1, cell adhesion, including Vcam, vacuolar transport, including RhoB and the MAPK pathway, including Spred1, were shown to be downregulated in splenic $\mathrm{T}$ cells isolated from animals treated with allochimeric MHC compared with rejecting controls [58]. Since all these genes play a role in cell polarity and motility we proposed that the early immunosuppressive effects attributed to allochimeric MHC treatment was related to the impairment of T cell motility, immunological synapse formation, and ability of $\mathrm{T}$ cells to infiltrate the allograft (Figures 4-5) [57,58].

We further investigated the role of RhoA, a member of GTPase protein family, in splenic T cells and its contribution to the attenuation of chronic rejection. Using a combination of RT-PCR and Western blot analysis, we found that $\mathrm{T}$ cell expression of RhoA was significantly reduced following allochimeric $\mathrm{MHC}$ treatment compared with untreated and CsA-treated controls [59]. This finding suggested that the early post-transplantation inhibition of RhoA played a role in diminishing the early immune response against a foreign graft. In fact, immunostaining for RhoA showed aberrant RhoA distribution within splenic $\mathrm{T}$ cells in the allochimeric MHC-treated animals isolated at day 1 and 3 post-transplantation compared with CsA-treated controls [59]. Given the role dysregulation.of RhoA in cytoskeleton organization and actin filament polymerization, we further showed that distribution of actin was highly disorganized in $\mathrm{T}$ cells from the allochimeric MHC-treated cohort (Figure 6) [59]. In addition, actin binding partner, protein Hip55 was partially dislocated from the actin in allochimeric treated T cells (Figure 7) [59]. We suggested that disruption of the $\mathrm{T}$ cell cytoskeleton led to the impairment of $\mathrm{T}$ cell migration toward its target, whether it is an APC or foreign protein antigen (Figures 4-5) [59]. In fact, histology of allografts from animals treated with allochimeric MHC showed significantly reduced $\mathrm{T}$ cell infiltration into allografts compared to rejecting controls [59].

Another possible mechanism underlying the immunosuppressive properties of soluble $\mathrm{MHC}$ is the way that this protein molecule is processed by antigen-presenting cells, such as the DCs. Through an elaborate multistep process involving vesicular trafficking between the endoplasmic reticulum (ER) and the Golgi apparatus, protein antigens are displayed to peripheral $\mathrm{T}$ cells, and an immune response is executed. However, nothing is known about how allochimeric class I MHC is processed

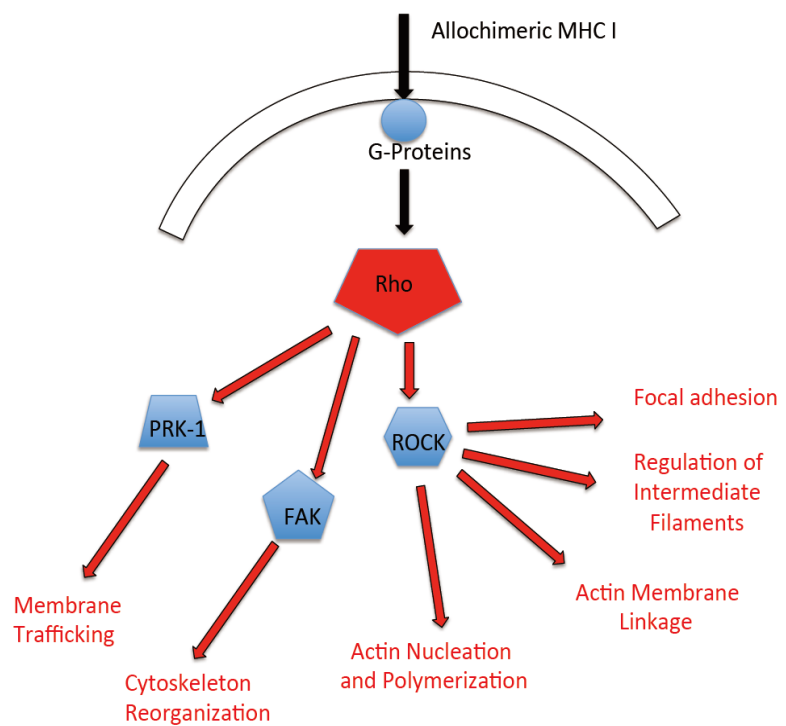

Rho GTPase pathway related cell functions and events (marked in red) potentially inhibited by allochimeric MHC I treatment in T cells and dendritic cells in rat cardiac allograft model system (see text).

Figure 5. Molecular and cellular targets of Rho pathway.

within the DC and presented to T cells. Looking at the vesicular trafficking pathway and the morphological appearance of the ER and Golgi apparatus, we discovered stark differences in DCs isolated from allochimeric MHC treated recipients compared with untreated and CsA-treated controls [36]. We also found that there was decreased expression and abnormal distribution of RhoB in DCs from animals treated with allochimeric MHC [36]. Endocytic/intracellular membrane trafficking relies on RhoB [60] and its decreased expression compared with controls would indicate that the earliest step in antigen processing has been dysregulated [36]. Furthermore, looking at the ER and Golgi resident proteins KDEL and GM130, we found distinctly abnormal morphology of these two organelles in DCs from allochimeric MHC treated animals. We found that the ER lost its vesicular appearance and dissociated from its normal paranuclear localization. The Golgi apparatus also lost much of its vesicular appearance and became markedly reduced in size compared to controls [36]. These changes suggest that in our model system, the intracellular processing of soluble antigen, such as allochimeric MHC, has been highly disregulated (Figures 2-3).

Our previous and recent studies indicate that intra-operative treatment with allochimeric class I MHC attenuates chronic rejection. Chronic rejection is characterized by perivascular graft inflammation, graft vascular intimal hyperplasia and progressive luminal narrowing, as well as necrotizing arteritis. These lesions were shown by Singer et al. [38] to be diminished in long-term graft-surviving ACI recipients of WF grafts compared with 

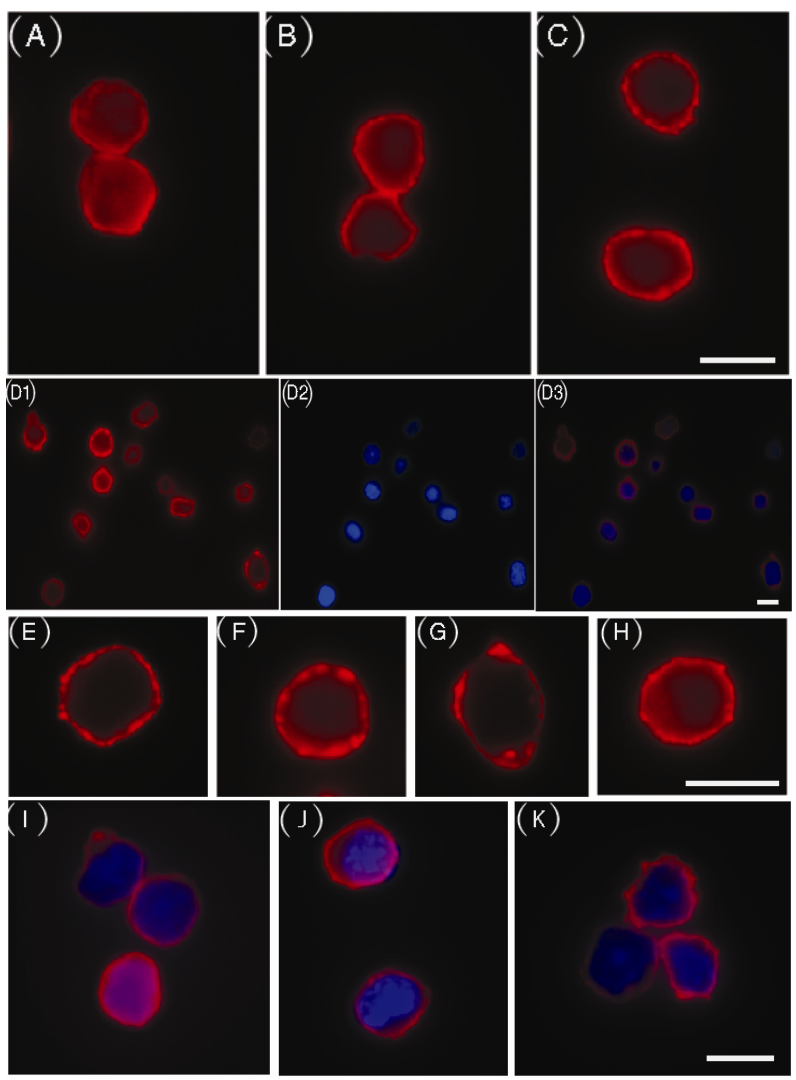

(A-C, D1, D3, E-K) Rhodamine-phalloidin staining (red) of actin in T cells isolated from spleen. (D2, D3, I-K) T cell nuclei are counterstained with Hoechst (blue). (D3, I-K) Merged images of actin (red) and nuclear (blue) Hoechst staining. (A) T cells from control,untreated animals at day 1 and (I) at day 3 post-transplantation. (A, I) Uniform layer of cortical actin is visible underneath the cellular membrane. (B) $\mathrm{T}$ cells from animals treatedwith a sub-therapeutic dose of $\mathrm{CsA}$ at day 1 and $(\mathrm{J})$ at day 3 post-transplantation. $(\mathrm{B}, \mathrm{J})$ Actin distribution becomes patchy; small aggregates of actin underlie the cellular membrane. (D1-H) T cells from animals treated with a sub-therapeutic dose of CsA in conjunction with allochimeric molecule at day 1 and $(\mathrm{J})$ at day 3 post-transplantation. Actin forms large aggregates unevenly distributed below the cellular membrane. Bar is equal to $10 \mu \mathrm{m}$. (Figure originally published in Skelton et al. Transplant Immunology 23 (2010) 185-193; with the permission from Elsevier).

Figure 6. Allochimeric molecule treatment induces changes in cortical actin distribution in $\mathrm{T}$ cells.

untreated and CsA-treated controls. In addition, the adoptive transfer of $\mathrm{T}$ cells conditioned with allochimeric class I MHC into secondary recipients also resulted in decreased evidence of chronic rejection, which suggests the role of antigen-specific $\mathrm{T}$ regulatory cells in attenuating detrimental graft injury [61].

Although further studies are necessary to fully understand the role of MHCs in the attenuation of chronic rejection, our studies indicate that the development of tolerance is multifactorial. We believe that perioperative exposure to donor-specific epitopes of class I MHC alters the recipient $\mathrm{T}$ cell and $\mathrm{DC}$ subcellular and Rho pathway related events (Figure 5). Thus, the effector function of recipient $\mathrm{T}$ cells and DCs is reduced, leading
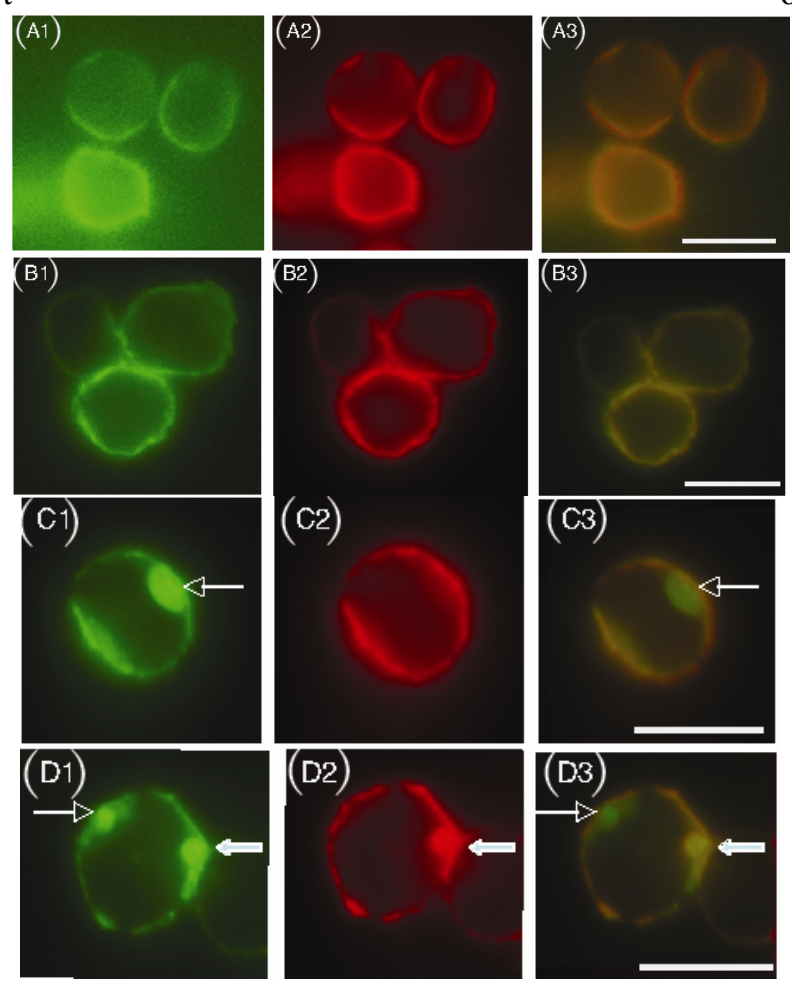

(A1, B1, C1 and D1) Immunostaining of T cells with anti-Hip55 antibody and FITC-conjugated secondary antibody (green) at day 1 post-transplantation. (A2, B2, C2, D2) Rhodamine-phalloidin staining (red) of actin in T cells. (A3, B3, C3, D3) Merged images of actin (red) and Hip55 (green) staining. (A1-A3) T cells from control, untreated animals. A uniform layer of cortical actin is visible underneath the cellular membrane and colocalizes with the actin-binding protein, Hip55. (B1-B3) T cells from animals treated with a sub-therapeutic dose of CsA. Actin and Hip55 distribution becomes patchy; small aggregates (on average 21 aggregates per cell), containing colocalized actin and Hip55, underlie the cellular membrane. (C1-D3) T cells from animals treated with a sub-therapeutic dose of CsA in conjunction with allochimeric molecule. Actin and Hip55 form large aggregates unevenly distributed below the cellular membrane. Some of these aggregates show colocalization of actin and Hip55 (thick arrows), and some Hip55-positive aggregates (on average 2.3 aggregates per cell) do not contain actin (thin arrows), which indicates that actin partially dissociates from its binding partner, Hip55. Bar is equal to $10 \mu \mathrm{m}$. (Figure originally published in Skelton et al.. Transplant Immunology 23 (2010) 185-193; with the permission from Elsevier).

Figure 7. Dissociation of actin-binding adaptor protein Hip55 from actin in $\mathrm{T}$ cells from allochimeric molecule-treated rats.

to the suppression of the recipient's immune response and the maintenance of immunological tolerance.

\section{ACKNOWLEDGMENTS}

We acknowledge NIH Grant RO1 AI49945 support to R. M. Ghobrial.

\section{REFERENCES}

[1] Bach, F.H. and Amos, D.B. (1967) Hu-1: Major histocompatibility locus in man. Science, 156, 1506-1508. doi:10.1126/science.156.3781.1506 
[2] Chinen, J. and Buckley, R.H. (2010) Transplantation immunology: Solid organ and bone marrow. Journal of Allergy and Clinical Immunology, 125, S324-S335. doi:10.1016/j.jaci.2009.11.014

[3] Cohen, D.J., Loertscher, R., Rubin, M.F., Tilney, N.L., Carpenter, C.B. and Strom, T.B. (1984) Cyclosporin: A new immunosuppressive agent for organ transplantation. Annals of Internal Medicine, 10, 667-682.

[4] Hariharan, S., Johnson, C.P., Bresnahan, B.A., et al. (2000) Improved graft survival after renal transplantation in the United States, 1988 to 1996. The New England Journal of Medicine, 342, 605-612. doi:10.1056/NEJM200003023420901

[5] Cecka, J.M. (2002) The UNOS Renal Transplant Registry. Clinical Transplantation, 1-20.

[6] Safinia, N., Afzail, B., Atalar, K., et al. (2010) T-cell alloimmuntiy and chronic allograft dysfunction. Kidney international, 78 (suppl 119), S2-S12. doi:10.1038/ki.2010.416

[7] Klein, J. and Sato, A. (2000) The HLA system: Second of two parts. The New England Journal of Medicine, 343, 782-786.

[8] Marsh, S.G. (2009) WHO nomenclature committee for factors of the HLA system. Nomenclature for factors of the HLA system, update. Tissue Antigens, 74, 364-366. doi:10.1111/j.1399-0039.2009.01330.x

[9] Klein, J. and Sato, A. (2000) The HLA system: First two parts. The New England Journal of Medicine, 343, 702-709.

[10] Zinkernagel, R.M. and Doherty, P.C. (1974) Restriction of invitro $\mathrm{T}$ cell-mediated cytotoxicity in lymphocytic choriomeningitis within a syngeneic or semiallogeneic system. Nature, 248, 701-702. doi:10.1038/248701a0

[11] Scott, A., Sant, L. and Sant, A. (2010) Generation of MHC class II-peptide ligands for CD4 T cell allorecognition of MHC class II molecules. CurrOpin Organ Transplant, 15, 505-511. doi:10.1097/MOT.0b013e32833bfc5c

[12] Li, X. and Raghavan, M. (2010) Structure and function of major histocompatability complex class I antigens. CurrOpin Organ Transplant, 15, 499-504. doi:10.1097/MOT.0b013e32833bfb33

[13] Trivedi, H.L. (2007) Immunobiology of rejection and adaptation. Transplantation Proceedings, 39, 647-652. doi:10.1016/j.transproceed.2007.01.047

[14] Stern, L.J. and Wiley, D.C. (1994) Anitgenic peptide binding by class I and class II histocompatibility proteins. Structure, 2, 245-251. doi:10.1016/S0969-2126(00)00026-5

[15] Jensen, P.E. (2007) Recent advances in antigen processing and presentation. Nature Immunology, 8, 1041-1048. doi:10.1038/ni1516

[16] Afzail, B., Lombardi, G. and Lechler, R. (2008) Pathways of major histocompatability complex allorecognition. Current Opinion in Organ Transplantation, 13, 438-444. doi:10.1097/MOT.0b013e328309ee31

[17] Jensen, P.E. (1999) Mechanisms of antigen presentation.
Clinical Chemistry and Laboratory Medicine, 37, 179-186. doi:10.1515/CCLM.1999.034

[18] Elliot, T. and Williams, A. (2005) The optimization of peptide cargo bound to MHC class I molecules by the peptide-loading complex. Immunological Reviews, 207, 89-99. doi:10.1111/j.0105-2896.2005.00311.x

[19] Warrens, A.N., Lombardi, G., Lechler, R.I., et al. (1994) Presentation and recognition of major and minor histocompatability antigens. Transplant Immunology, 2, 103-107. doi:10.1016/0966-3274(94)90036-1

[20] Lechler, R.I. and Batchelor, J.R. (1982) Restoration of immunogenicity to passenger cell-depleted kidney allografts by the addition of donor strain dendritic cells. The Journal of Experimental Medicine, 155, 31-41. doi:10.1084/jem.155.1.31

[21] Ely, L.K., Burrows, S.R., Purcell, A.W., et al. (2008) T cells behaving badly: Structural insights into alloreactivity and autoimmunity. Current Opinion in Organ Transplantation, 20, 575-580. doi:10.1016/j.coi.2008.07.006

[22] Baker, R.J., Hernandez-Fuentes, M.P., Brookes, P.A., et al. (2001) The role of the allograft in the induction of donor-specific T cell hyporesponsiveness. Transplantation, 72, 480-485. doi:10.1097/00007890-200108150-00020

[23] Suchin, E.J., Langmuir, P.B., Palmer, E., et al. (2001) Qunatifying the frequency of allreactive $\mathrm{T}$ cells in vivo: new answeres to an old question. The Journal of Immunology, 166, 973-981.

[24] Gras, S., Kjer-Nelson, L., Chen, Z., et al. (2011) The structural bases of direct T-cell allorecognition: Implication for T-cell mediated transplant rejection. Immunology and Cell Biology, 5, 1-8.

[25] Crispe, I.N., Husmann, L.A. and Bevan, M.J. (1986) T cell receptor expression and receptor-mediated induction of clonal growth in the developing mouse thymus. High surface beta-chain density is a requirement for functional maturity. European Journal of Immunology, 16, 1283-1288. doi:10.1002/eji.1830161016

[26] Matzinger, P. and Bevan, M.J. (1977) Hypothesis: Why do so many lymphocytes respond to major histocompatability antigens? Cell Immunology, 29, 1-5. doi:10.1016/0008-8749(77)90269-6

[27] Archbold, J.K., Macdonald, W.A., Miles, J.J., et al. (2006) Alloreactivity between disparate cognate and allogeneic pMHC-I complexes is the result of highly focused, peptide-dependent structural mimicry. The Journal of Biological Chemistry, 281, 34324-34332. doi:10.1074/jbc.M606755200

[28] Turner, S.J., Doherty, P.C., McClusky, J., et al. (2006) Structural derminants of T-cell receptor bias in immunity. Nature Reviews Immunology, 6, 883-894. doi:10.1038/nri1977

[29] Pietra, B.A., Wiseman, A., Bolwerk, A., et al. (2000) CD4 $\mathrm{T}$ cell-mediated cardiac allograft rejection requires donor but not host MHC class II. The Journal of Clinical Investigation, 106, 1003-1010.

[30] Shoskes, D.A. and Wood, K.J. (1994) Indirect presenta- 
tion of MHC antigens in transplantation. Immunology Today, 15, 32-38. doi:10.1016/0167-5699(94)90023-X

[31] Liu, Z., Braunstein, N.S. and Suciu, F.N. (1992) T cell recognition of allopeptides in context of self $\mathrm{MHC}$. The Journal of Immunology, 148, 35-40.

[32] Watts, C. (2004) The exogenous pathway for antigen presentation on major histocompatibility complex class II and CD1 molecules. Nature Immunology, 5, 670-677. doi:10.1038/ni1088

[33] Cresswell, P. (1996) Invariant chain structure and MHC class II function. Cell, 84, 505-507. doi:10.1016/S0092-8674(00)81025-9

[34] Inaba, K., Turley, S., Yamaide, F., et al. (1998) Efficient presentation of phagocytosed cellular fragments on the major histocompatibility complex class II products of dendritic cells. The Journal of Experimental Medicine, 188, 2163-2173. doi:10.1084/jem.188.11.2163

[35] Fangmann, J., Dalchau, R. and Fabre, J.W. (1992) Rejection of skin allografts by indirect allorecognition of donor class I major histocompatabiltiy complex peptides. The Journal of Experimental Medicine, 175, 1521-1529. doi:10.1084/jem.175.6.1521

[36] Skelton, T.S., Tejpal, N., Gong, Y., et al. (2011) Allochimerich molecules and mechanisms in the abrogation of cardiac allograft rejection. The Journal of Heart and Lung Transplantation, Epub, ahead of print.

[37] Gokmen, M.R., Giovanna, L. and Lechler, R.I. (2008) The importance of the indirect pathway of allorecognition in clinical transplantation. Current Opinion in Immunology, 20, 568-574. doi:10.1016/j.coi.2008.06.009

[38] Singer, J.S., Mhoyan, A., Fishbein, M., et al. (2001) Allochimeric class I MHC molecules prevent chronic rejection and attenuate alloantibody response. Transplantation, 72, 1408-1416. doi:10.1097/00007890-200110270-00014

[39] Valujskikh, A., Lantz, O., Celli, S., et al. (2002) Cross primed $\mathrm{CD}^{+} \mathrm{T}$ cells mediate graft rejection via a distinct effector pathway. Nature Immunology, 3, 844-851. doi:10.1038/ni831

[40] Kapessidou, Y., Habran, C., Buonocore, S., et al. (2006) The replacement of graft endothelium by recipient-type cells conditions allograft rejection mediated by indirect pathway $\mathrm{CD}^{+} \mathrm{T}$ cells. Transplantation, 82, 582-591. doi:10.1097/01.tp.0000184444.93108.d1

[41] Herrera, O.B., Golshayan, D., Tibbott, R., et al. (2004) A novel pathway of alloantigen presentation by dendritic cells. The Journal of Immunology, 173, 4828-4837.

[42] Ridge, J.P., Di, R.F. and Matzinger, P.A. (1998) A conditioned dendritic cell can be a temporal bridge between a $\mathrm{CD}^{+}$T-helper and a T-killer cell. Nature, 393, 474-478. doi:10.1038/30989

[43] Lee, R.S., Grusby, M.J., Glimcher, L.H., et al. (1994) Indirect recognition by helper cells can induce donor-specific cytotoxic T lymphocytes in vivo. The Journal of Experimental Medicine, 179, 865-872. doi:10.1084/jem.179.3.865

[44] Wise, M.P., Bemelman, F., Cobbold, S.P., et al. (1998) Linked suppression of skin graft rejection can operate through indirect recognition. The Journal of Immunology, 161, 5813-5816.

[45] Games, D.S., Rogers, N.J. and Lechler, R.I. (2005) Acquisition of HLA-DR and costimulatory molecules by $\mathrm{T}$ cells from allogeneic antigen presenting cells. American Journal of Transplantation, 5, 1614-1625. doi:10.1111/j.1600-6143.2005.00916.x

[46] Morelli, A.E., Larregina, A.T., Shufesky, W.J., et al. (2004) Endocytosis, intracellular sorting and processing of exosomes by dendritic cells. Blood, 104, 3257-3266. doi:10.1182/blood-2004-03-0824

[47] Pimenta-Araujo, R., Mascarell, L., Huesca, M., et al. (2001) Embryonic thymic epithelium naturally devoid of APCs is acutely rejected in the absence of indirect recognition. The Journal of Immunology, 167, 5034-5041.

[48] Calne, R.Y., Sells, R.A., Pena, J.R., et al. (1969) Induction of immunologic tolerance by porcine liver allografts. Nature, 223, 472-476. doi:10.1038/223472a0

[49] Zeigler, S.F. (2006) Foxp3: Of mice and men. Annual Review of Immunology, 24, 209-226. doi:10.1146/annurev.immunol.24.021605.090547

[50] Toyokawa, H., Nakao, A., Bailey, R.J., Nalesnik, M.A., et al. (2008) Relative contribution of direct and indirect allorecognition in developing tolerance after liver transplantation. Liver Transplantation, 14, 346-357. doi:10.1002/lt.21378

[51] Callaghan, C.J., Rouhani, F.J., Negus, M.C., Curry, A.J., Bolton, E.M., Bradley, J.A. and Pettigrew, G.J. (2007) Abrogation of antibody-mediated allograft rejection by regulatory $\mathrm{CD} 4 \mathrm{~T}$ cells with indirect allospecificity. The Journal of Immunology, 178, 2221-2228.

[52] Billingham, R.E., Brent, L. and Medawar, P.B. (2010) Actively acquired tolerance of foreign cell. 1953. The Journal of Immunology, 184, 5-8. doi:10.4049/jimmunol.0990109

[53] Sayegh, M.H., Perico, N., Gallon, L., et al. (1994) Mechanism of acquired thymichyporesponsiveness to renal allografts. Thymic recognition of immunodominantallo-MHC peptides induces peripheral T cell anergy. Transplantation, 58, 125-132. doi:10.1097/00007890-199407270-00001

[54] Zhou, C., Lu, R., Lin, G., et al. (2011) The latest developments in synthetic peptides with immunoregulatory activities. Peptides, 32, 408-414. doi:10.1016/j.peptides.2010.10.019

[55] Krensky, A.M. and Clayberger, C. (1997) HLA-derived peptides as novel immunosuppressives. Nephrology Dialysis Transplantation, 12, 865-878. doi:10.4049/jimmunol.0990109

[56] Gobrial, R.M., Hamashima, W., Wang, M., et al. (1996) Induction of transplantion tolerance by chimeric donor/recipient class I RT1. $\mathrm{A}^{\mathrm{a}}$ molecules. Transplantation, 62, 1002-1010. doi:10.1097/00007890-199610150-00020

[57] Lisik, W., Gong, Y., Tejpal, N., et al. (2010) Intragraft gene expression profile associated with the induction of tolerance by allochimeric MHC I in the rat heart transplant model. Genesis, 1, 8-19. 
[58] Lisik, W., Tejpal, N., Gong, Y., et al. (2009) Down regulation of genes involved in $\mathrm{T}$ cell polarity and motility during the induction of heart allograft tolerance by allochimeric MHC I. PlosOne, 4, e8020. doi:10.1371/journal.pone. 0008020

[59] Skelton, T.S., Tejpal, N., Gong, Y., et al. (2010) Downregulation of RhoA and changes in $\mathrm{T}$ cell cytoskeleton correlate with the abrogation of allograft rejection. Transplant Immunology, 23, 185-193. doi:10.1016/j.trim.2010.06.009
[60] Wheeler, A.P. and Ridley, A.J. (2004) Why three Rho proteins? RhoA, RhoB, RhoC and cell motility. Experimental Cell Research, 301, 43-49. doi:10.1016/j.yexcr.2004.08.012

[61] Semiletova, N., Shen, X.D., Baibakov, B., et al. (2010) Intensity of transplant chronic rejection correlates with level of graft-infiltrating regulatory cells. The Journal of Heart and Lung Transplantation, 29, 335-341. doi:10.1016/j.healun.2009.08.003 